07

\title{
Особенности работы мощных AllnGaN-светодиодов при больших импульсных токах
}

\author{
(ㄱ А.Л. Закгейм ${ }^{1}$, А.Е. Иванов ${ }^{1,2}$, А.Е. Черняков ${ }^{1}$ \\ ${ }^{1}$ НТЦ микроэлектроники РАН, Санкт-Петербург, Россия \\ ${ }^{2}$ Санкт-Петербургский государственный электротехнический университет „ЛЭтИ“, Санкт-Петербург, Россия \\ E-mail: zakgeim@mail.ioffe.ru
}

Поступило в Редакцию 31 марта 2021 г.

В окончательной редакции 30 апреля 2021 г.

Принято к публикации 11 мая 2021 г.

\begin{abstract}
Исследованы токовые зависимости мощностных и спектральных характеристик, в том числе их распределение (мэппинг) по излучающей поверхности, AlInGaN-светодиодов „вертикальной“ конструкции в широком диапазоне рабочих токов вплоть до $\sim 70 \mathrm{~A}$. Установлено, что начиная с определенного уровня возбуждения некорректно использовать представление о средней плотности тока при анализе падения эффективности. Главным фактором снижения внутреннего квантового выхода и коэффициента вывода излучения, ограничивающим энергетические возможности светодиода, становится эффект шнурования („current crowding“) тока к контактам.
\end{abstract}

Ключевые слова: AlInGaN-светодиод, квантовый выход, спектр излучения, ближнее поле излучения.

DOI: 10.21883/PJTF.2021.16.51326.18795

AlInGaN-светодиоды сине-зеленого диапазона излучения наиболее массово используются в светотехнике, т. е. работают „на человеческий глаз“. В связи с этим большинство исследований мощностных и спектральных характеристик, в том числе и хорошо известного эффекта падения эффективности с током „efficiency droop“ $[1,2]$, проводилось в режиме либо постоянного тока, либо близкого к нему — в режиме широтно-импульсной модуляции. В последнее время возрос интерес к использованию AlInGaN-светодиодов видимого диапазона для работы с физическими приемниками, например, в системах VLC (visible light communication) или для накачки твердотельных лазеров с малым временем жизни активатора (Ті: сапфир) [3]. Новые приложения требуют работы светодиодов в режимах коротких импульсов (десятки наносекунд-единицы микросекунд) при достижении максимальной мощности излучения (энергии в импульсе). Настоящая работа посвящена исследованию таких режимов и выявлению причин, ограничивающих энергетические возможности светодиодов.

Исследования проводились на коммерческих светодиодах SemiLEDs EV-B40A „вертикальной“ конструкции [4] площадью $\sim 1 \mathrm{~mm}^{2}$ со сплошным нижним контактом и полосковым верхним (вставка на рис. 1,a), сохраняющим работоспособность до токов $I \sim 70$ А при длительности импульсов до $\tau \sim 3 \mu \mathrm{s}$. На рис. $1, a$ приведены токовые зависимости мощности излучения $P_{\text {opt }}$ и внешнего квантового выхода $\eta_{\mathrm{EQE}}$, а на рис. $1, b-$ пиковой длины волны излучения $\lambda_{\text {peak }}$ и ширины спектра излучения на уровне половины максимума (полуширины) $\Delta \lambda_{0.5}$ в широком диапазоне токов, перекрывающем пять порядков: от долей миллиампера до 70 А. Импульсный режим при измерениях $(\tau=100 \mathrm{~ns}, F=100 \mathrm{~Hz})$ обеспечивался генератором Agillent 8114A с усилителем
PicoLAS LDP-V 80-100 V3.3. Оптическая мощность и спектры излучения регистрировались комплексом „OL 770-LED High-speed LED Test and Measurement System“, температура образца $T=300 \mathrm{~K}$.

Как видно из рис. 1, можно выделить две области: 1) примерно до $10 \mathrm{~A} \mathrm{c} \mathrm{резким} \mathrm{изменением} \mathrm{квантового}$ выхода и спектральных характеристик; 2) более 10 А, где указанные характеристики асимптотически стремятся к некоторым постоянным значениям.

Для моделирования вида зависимостей, приведенных на рис. $1, a$, нами привлекалась $A B C$-модель, в которой внутренний квантовый выход определяется конкуренцией трех механизмов рекомбинации в активной области [1]:

$$
\eta_{\mathrm{EQE}}=\eta_{\text {ext }} \eta_{\mathrm{IQE}}=\eta_{\text {ext }} \frac{B n^{2}}{A n+B n^{2}+C n^{3}} .
$$

Здесь $\eta_{\mathrm{IQE}}-$ внутренний квантовый выход, $\eta_{\text {ext }}-$ коэффициент вывода излучения, $n-$ концентрация носителей, $A, B$ и $C-$ коэффициенты рекомбинации по Шокли-Риду-Холлу, бимолекулярной излучательной рекомбинации и оже-рекомбинации, соответственно. С использованием известных преобразований [5] при построении экспериментальных зависимостей приведенного квантового выхода $\eta_{\mathrm{EQE}}^{\max } / \eta_{\mathrm{EQE}}$ от суммы корней приведенных мощностей $\left(p^{1 / 2}+p^{-1 / 2}\right)$, где $p=P_{\text {out }} / P_{\text {out }}^{\max }$, а $P_{\text {out }}^{\max }$ - выходная оптическая мощность при токе, соответствующем $\eta_{\mathrm{EQE}}^{\max }$, получаем выражение для определения основных параметров излучательной рекомбинации:

$$
\eta_{\mathrm{EQE}}^{\max } / \eta_{\mathrm{EQE}}=\eta_{\mathrm{IQE}}^{\max }+\frac{p^{1 / 2}+p^{-1 / 2}}{Q+2},
$$

где $Q=B /(A C)^{1 / 2}$ — „фактор качества“. Из графика на основе (2) при его экстраполяции к значению 


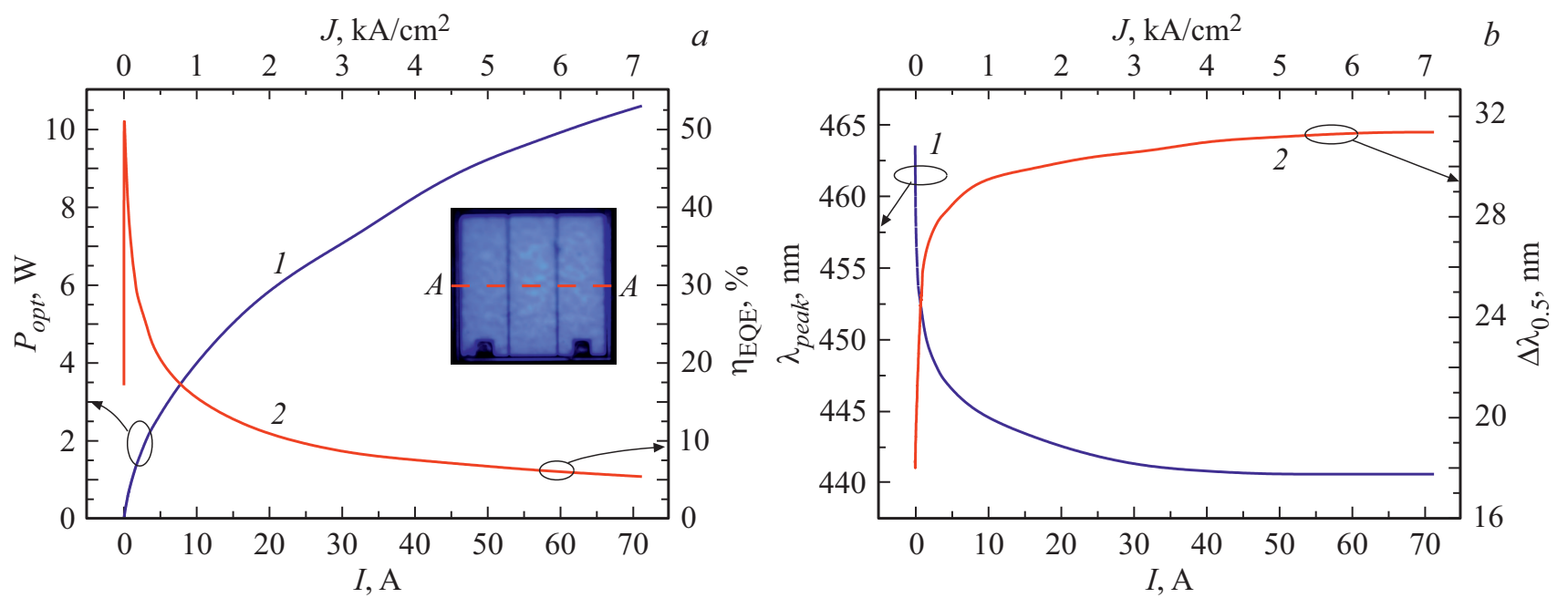

Рис. 1. Зависимости от тока: $a) 1-$ выходной оптической мощности $P_{\text {out }}, 2-$ внешнего квантового выхода $\eta_{\mathrm{EQE}}$; $\left.b\right) 1-$ пиковой длины волны $\lambda_{\text {peak }}, 2-$ полуширины спектра $\Delta \lambda_{0.5}$. На вставке - фотография излучающего кристалла.

$\left(p^{1 / 2}+p^{-1 / 2}\right) \rightarrow 0$ можно определить $\eta_{\mathrm{IQE}}^{\max }$, а из наклона $-Q$. С учетом $\eta_{\mathrm{EQE}}=\eta_{\text {ext }} \eta_{\mathrm{IQE}}$, зная $\eta_{\mathrm{IQE}}$ и экспериментально измеренный $\eta_{\mathrm{EQE}}$, можно определить и $\eta_{\text {ext }}$, т.е. все главные параметры светодиода. Результаты соответствующего построения зависимости $\eta_{\mathrm{EQE}}^{\max } / \eta_{\mathrm{EQE}}$ от комбинации $\left(p^{1 / 2}+p^{-1 / 2}\right)$ на основе экспериментальной зависимости (рис. $1, a$ ) на участке умеренных токов $(0.1-0.5 \mathrm{~A})$ и их анализа дали значения $\eta_{\mathrm{IQE}}^{\max }=0.83$, $Q=10, \eta_{\text {ext }}=0.61$.

На рис. 2, а представлены расчетная зависимость $\eta_{E Q E}=f(I)$ по $A B C$-модели, экстраполированная до тока 70 А (кривая 1), и аналогичная экспериментальная зависимость (кривая 2). Значительное расхождение кривых, начиная с токов в единицы ампер, требует объяснения, в качестве которого мы предположили неправомочность представления о равномерности распределения тока по площади $p-n$-перехода при его возрастании до больших значений (т.е. использования в расчетах значения средней плотности тока $J=I / S$, где $I-$ ток, $S$ - площадь кристалла). Для проверки указанного предположения и количественной оценки распределения плотности тока нами исследовалось ближнее поле излучения на верхней грани кристалла, включая как интенсивность, так и спектр излучения. Мощностной и спектральный мэппинг осуществлялся с помощью оптического микроскопа Mitutoyo, совмещенного со спектрометром Avantes AvaSpec-2048. Оптическая система обеспечивала пространственное разрешение $\sim 30 \mu \mathrm{m}$.

На рис. 2, $b$ представлено экспериментальное распределение интенсивности излучения в зависимости от координаты $x$ - удаления от края контактной площадки (расстояние между контактами $280 \mu \mathrm{m}$ ). Как видно из pис. 2, $b$, заметное отклонение от однородного распределения начинается при токах в единицы ампер, далее неоднородность нарастает, и при токе 70 А значение интенсивности излучения в середине между контактами составляет $\sim 50 \%$ от максимальной величины (у края контакта). Следует отметить, что распределение интенсивности лишь качественно отражает распределение плотности тока. В силу главной причины - зависимости квантового выхода от тока, а также отчасти за счет рассеяния света внутри кристалла оно является более сглаженным по сравнению с распределением плотности тока [6]. Для дальнейшего уточнения распределения тока, а также температуры по площади кристалла мы использовали мэппинг спектрального распределения. На рис. 3, $a$ показаны нормированные спектры излучения при токе 70 А для двух точек поверхности кристалла: вблизи контакта (1) и посередине между контактами (2), а на рис. $3, b-$ смещение пиковой длины волны излучения $\lambda_{\text {peak }}$ при движении излучающей точки от края контакта к центру для разных токов в сечении $A A$ (вставка на рис. 1, $a$ ). Как видно из рис. 3, $a$, наклон коротковолнового плеча спектра не зависит от положения точки излучения (вблизи контакта или при удалении от него), что свидетельствует об однородности температуры по площади кристалла [7]. В то же время для тока 70 А наблюдается заметный коротковолновый сдвиг спектра, излучаемого вблизи котактной площадки $(x=0)$, по сравнению со спектром, излучаемым в середине между контактными площадками $(x=140)$ : $\lambda_{\text {peak }, 0 \rightarrow 140}=5 \mathrm{~nm}$, что является прямым подтверждением различия в плотностях тока. То же самое относится и к разнице в полуширинах спектра: $\Delta \lambda_{0.5,0 \rightarrow 140}=5 \mathrm{~nm}$. Оба показателя свидетельствуют о возникновении существенного градиента плотности тока при удалении от контактной площадки начиная с токов порядка единиц ампер.

Из сравнения „интегрального“ спектра излучения от всего кристалла с „точечными“ можно видеть, что при 70 А ,интегральный“ спектр по параметрам $\lambda_{\text {peak }}$ и $\Delta \lambda_{0.5}$ близок к „точечным“ из области, примыкающей к контакту. Последнее подтверждает, что в диапазоне больших токов основной вклад в излучение вносят области 
около контактов, причем там плотность тока настолько велика, что зависимость спектральных характеристик от нее практически отсутствует (достигает насыщения).

Приведенные экспериментальные данные свидетельствуют о следующем. Начиная с определенной величины токов, лежащей в области единиц ампер, становится некорректным рассматривать плотность тока как среднюю от деления величины тока на площадь кристалла и соответственно использовать ее в модельных расчетах. Эффективная плотность тока выше, и, следовательно, падение внутреннего квантового выхода $\eta_{\mathrm{IQE}} \mathrm{c}$ током сильнее предсказываемого расчетом по $A B C$-модели. К этому добавляется и уменьшение коэффициента вывода света $\eta_{\text {ext }}$ в связи со шнурованием тока вблизи контакта и увеличением эффекта затенения. В совокупности оба эффекта усугубляют процесс падения внешнего квантового выхода $\eta_{\mathrm{EQE}}$ при больших токах. С практической точки зрения учет выявленных эффектов означает необходимость особого подхода к конструированию
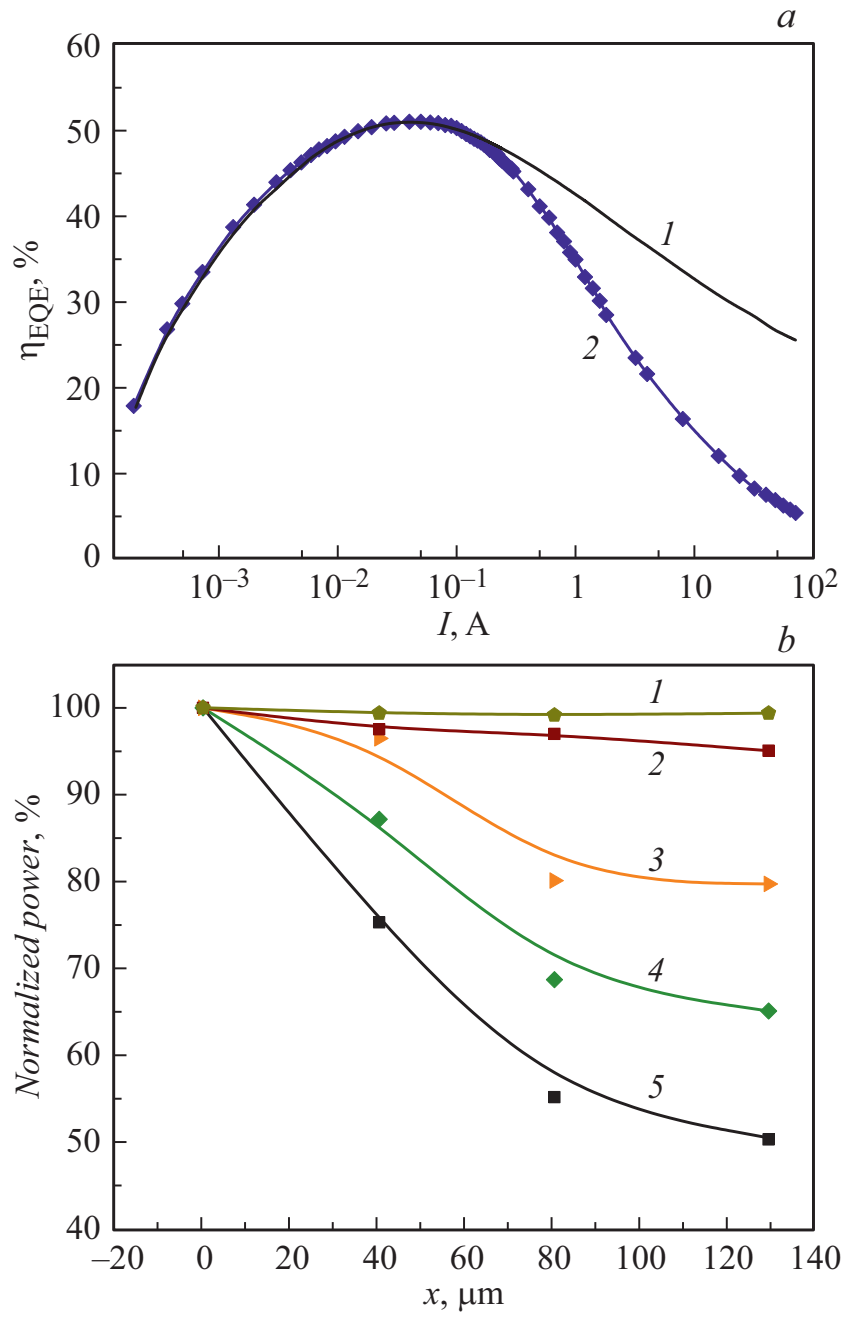

Pис. 2. $a-$ рассчитанная по $A B C$-модели зависимость $\eta_{\mathrm{EQE}}$ от тока (1) и экспериментальная зависимость (2); $b$ - распределение интенсивности излучения в зависимости от удаления от края контактной площадки при токах $I=0.1$ (1), 1 (2), 4 (3), 20 (4) и $70 \mathrm{~A}(5)$.
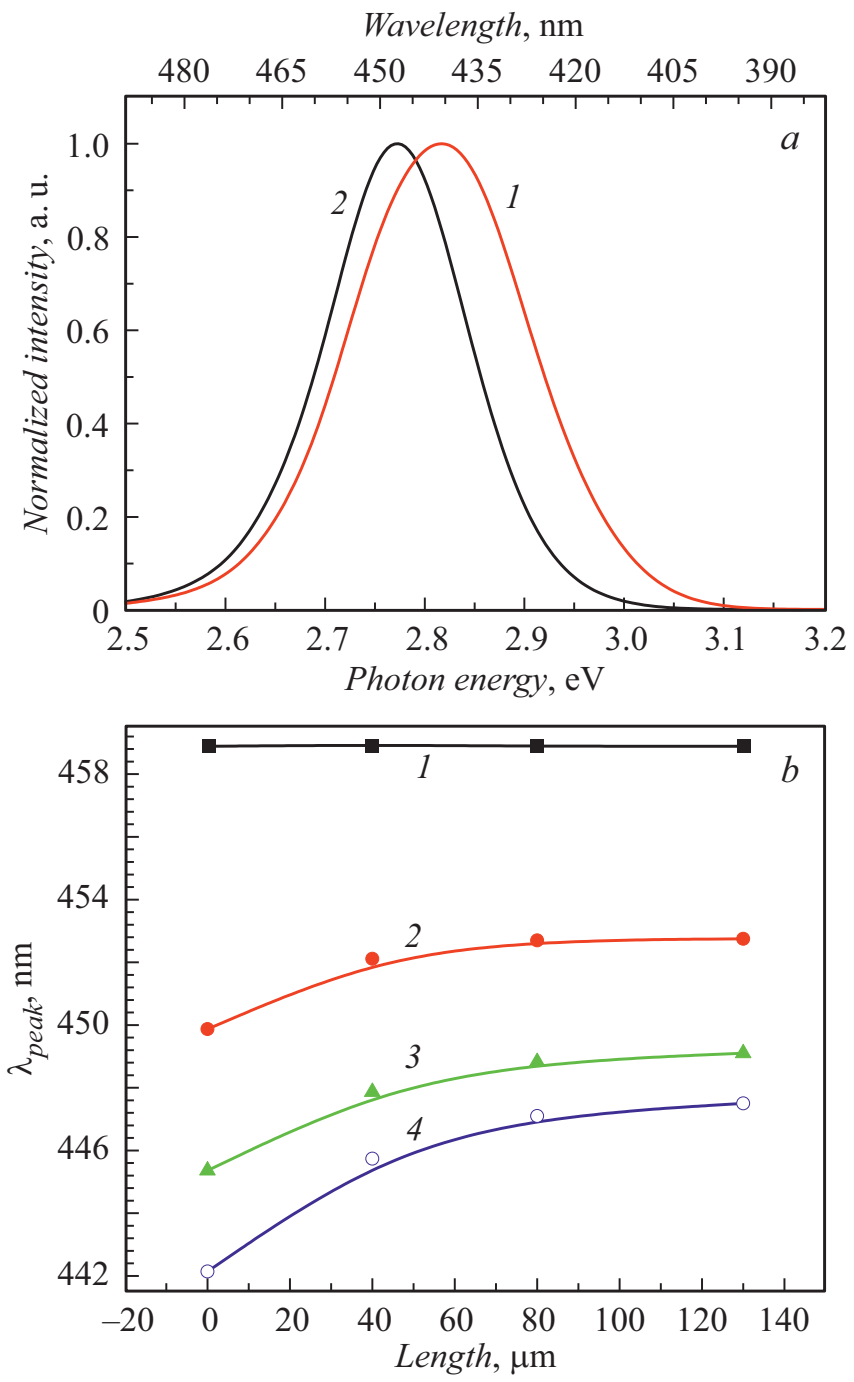

Рис. 3. $a-$ спектры излучения при токе $70 \mathrm{~A}$ в сечении $A A$ для двух точек: вблизи края контакта (1) и посередине между контактами $(2) ; b-$ зависимость $\lambda_{\text {peak }}$ от координаты (удаления от контакта) при токах $I=0.1(1), 4$ (2), 20 (3) и $70 \mathrm{~A}(4)$.

импульсных светодиодов, предназначенных для работы при больших токах. Топология котактов должна быть оптимизирована для обеспечения условий одородности растекания тока. Вероятно, это возможно при использовании сплошного прозрачного верхнего контакта или конструкций кристаллов с обоими тыльными контактами (типа „flip-сір“ [8] или „UX:3“ [9]), но требуются дальнейшие исследования.

\section{Благодарности}

Исследования параметров светодиодов выполнены в ЦКП „Элементная база радиофотоники и наноэлектроники: технология, диагностика, метрология“" 


\section{Конфликт интересов}

Авторы заявляют, что у них нет конфликта интересов.

\section{Список литературы}

[1] S.Yu. Karpov, Opt. Quant. Electron., 47 (6), 1293 (2015). DOI: $10.1007 / \mathrm{s} 11082-014-0042-9$

[2] Н.М. Шмидт, Е.И. Шабунина, А.Е. Черняков, А.Е. Иванов, Н.А. Тальнишних, А.Л. Закгейм, Письма в ЖТФ, 46 (24), 45 (2020). DOI: 10.21883/PJTF.2020.24.50429.18512

[3] P. Pichon, A. Barbet, J.-P. Blanchot, F. Druon, F. Balembois, P. Georges, Optica, 5 (10), 1236 (2018). DOI: $10.1364 /$ OPTICA.5.001236

[4] B. Hahn, B. Galler, K. Engl, Jpn. J. Appl. Phys., 53 (10), 100208 (2014). DOI: 10.7567/JJAP.53.100208

[5] I.E. Titkov, S.Yu. Karpov, A. Yadav, V.L. Zerova, M. Zulonas, B. Galler, M. Strassburg, I. Pietzonka, H. Lugauer, E.U. Rafailov, IEEE J. Quant. Electron., 50 (11), 911 (2014). DOI: $10.1109 /$ JQE.2014.2359958

[6] A.E. Chernyakov, K.A. Bulashevich, S.Yu. Karpov, A.L. Zakgeim, Phys. Status Solidi A, 210 (3), 466 (2013). DOI: $10.1002 /$ pssa.201200658

[7] A. Keppens, W.R. Ryckaert, G. Deconinck, P. Hanselaer, J. Appl. Phys., 108 (4), 043104 (2010). DOI: $10.1063 / 1.3463411$

[8] Д.А. Закгейм, Г.В. Иткинсон, М.В. Кукушкин, Л.К. Марков, О.В. Осипов, А.С. Павлюченко, И.П. Смирнова, А.Е. Черняков, Д.А. Бауман, ФТП, 48 (9), 1287 (2014).

[9] A. Laubsch, M. Sabathil, J. Baur, M. Peter, B. Hahn, IEEE Trans. Electron Dev., 57 (1), 79 (2010).

DOI: $10.1109 /$ TED.2009.2035538 